\title{
内蒙古不同类型草原土壤团聚体含量的分配及其 稳定性
}

\section{王 甜 ${ }^{1,2}$ 徐 姗 ${ }^{3}$ 赵梦颖 1,2 李 贺 ${ }^{1,2}$ 寇 丹 $^{1,2}$ 方精云 ${ }^{1}$ 胡会峰 ${ }^{*}$}

${ }^{1}$ 中国科学院植物研究所植被与环境变化国家重点实验室, 北京 100093 ; $^{2}$ 中国科学院大学, 北京 100049 ; $^{3}$ 中国科学院华南植物园, 广州 510650

摘 要 土壤团聚体是土壤结构的重要组成部分, 是土壤保护其有机碳的一种重要物理与生物机制, 但迄今为止对其空间格 局分布的研究较少。该文研究了我国内蒙古3种草原类型(草甸草原、典型草原、荒漠草原)不同土层深度的土壤团聚体质量 百分比及其稳定性的分布规律。结果显示: 土壤团聚体的质量百分比在3种草原类型各个土层深度的分布均呈现草甸草原> 典型草原=荒漠草原的趋势, 而沿土层深度 3 种草原类型的土壤团聚体的质量百分比含量并未呈现显著规律。各层的土壤团聚 体质量百分比均与年降水量呈显著正相关关系; 除70-100 cm 土层外, 其与年平均气温均呈负相关关系。对土壤团聚体稳定性 而言, 在0-10 cm 和10-20 cm两个土层深度, 草甸草原土壤团聚体的平均质量直径与几何平均直径显著大于典型草原和荒漠 草原, 而在其他土层, 3种草原类型间无显著差异。随着土层深度的增加, 草甸草原和典型草原土壤团聚体的平均质量直径与 几何平均直径均呈现逐渐降低的趋势。该文对于理解内蒙古不同类型草原土壤有机碳的稳定性和保护机制具有重要意义。 关键词 内蒙古草原; 土壤团聚体质量百分比; 土壤团聚体稳定性; 土层深度; 气候因子

引用格式: 王甜, 徐姗, 赵梦颖, 李贺, 寇丹, 方精云, 胡会峰 (2017). 内蒙古不同类型草原土壤团聚体含量的分配及其稳定性. 植物生态学报, 41, 1168-1176. doi: 10.17521/cjpe.2017.0220

\section{Allocation of mass and stability of soil aggregate in different types of Nei Mongol grasslands}

WANG Tian $^{1,2}$, XU Shan ${ }^{3}$, ZHAO Meng-Ying ${ }^{1,2}$, LI He ${ }^{1,2}$, KOU Dan $^{1,2}$, FANG Jing-Yun ${ }^{1}$, and HU Hui-Feng ${ }^{1 *}$

${ }^{1}$ State Key Laboratory of Vegetation and Environmental Change, Institute of Botany, Chinese Academy of Sciences, Beijing 100093, China; ${ }^{2}$ University of Chinese Academy of Sciences, Beijing 100049, China; and ${ }^{3}$ South China Botanical Garden, Chinese Academy of Sciences, Guangzhou 510650, China

\section{Abstract}

Aims Soil aggregate is an important component of soil structure, playing an important role in the physical and biological protection mechanism of soil organic carbon (SOC) through isolating SOC from microorganisms. As far as we know, there are few studies, however, on exploring the spatial distribution of soil aggregate at the regional scale. Our objective was to investigate the mass allocation and stability of soil aggregate in different types of Nei Mongol grasslands.

Methods We have established 78 sites with a size of $10 \mathrm{~m} \times 10 \mathrm{~m}$ across the transect of Nei Mongol grasslands and collected soil samples from different soil depth up to $1 \mathrm{~m}$. We used wet sieving method to separate different sizes of aggregate partition and used mean mass diameter (MMD) and geometric mean diameter (GMD) in order to evaluate the stability of soil aggregate. The two-way ANOVA was used to test the difference of mass percentage and stability of soil aggregate in different grassland types and soil depths. In addition, a linear regression analysis was used to analyze the correlations of mass percentage and stability of soil aggregate with both mean annual precipitation (MAP) and mean annual temperature (MAT).

Important findings The results showed that the mass percentages of soil aggregate were highest in meadow steppe, while almost equal in typical steppe and desert steppe. However, no significant patterns were found along the soil depth. The mass percentage of soil aggregate fractions were positively correlated with MAP in all soil layers, but negatively correlated with MAT except the layer of 70-100 cm. For the stability of soil aggregate, at 0-10 and 10-20 cm, MMD and GMD of meadow steppe were significantly greater than those of typical and desert steppes, whereas no significant differences among three grassland types were found for other soil layers. Besides, $M M D$ and $G M D$ in meadow steppe and typical steppe gradually decreased along the soil depth.

收稿日期Received: 2017-08-16 接受日期Accepted: 2017-11-14

* 通信作者Author for correspondence (E-mail: huifhu@ibcas.ac.cn) 
Key words Nei Mongol grasslands; mass percentage of soil aggregate; soil aggregate stability; soil depth; climate factors

Citation: Wang T, Xu S, Zhao MY, Li H, Kou D, Fang JY, Hu HF (2017). Allocation of mass and stability of soil aggregate in different types of Nei Mongol grasslands. Chinese Journal of Plant Ecology, 41, 1168-1176. doi: 10.17521/cjpe.2017.0220

土壤团聚体是土壤的重要组成部分, 对维持土 壤结构的稳定和土壤养分截留具有重要意义(Six et al., 1998; Cavagnaro et al., 2006)。此外, 土壤团聚体 对土壤有机碳具有物理保护作用，是土壤肥力的基 础和评价土壤质量的重要指标( $\mathrm{Lal}, 2000)$ 。表土中约 $90 \%$ 的有机质位于团聚体内(Jastrow，1996), 团聚体 将土壤有机碳与微生物隔离开, 从而保护有机碳难 被微生物分解以达到保护作用(Six et al., 2002a), 大 团聚体中包裹的小团聚体是土壤有机碳物理保护的 必要机制(Denef et al., 2007)。土壤团聚体是通过结 合体(如细根, 微生物菌丝, 糖分及黏粒、粉粒等)将 土壤颗粒粘结在一起而形成, 因此影响结合体形式 和量的因素均可能影响土壤团聚体的形成和分布 (Six et al., 2002b)。

草原是受人类活动影响最为严重的区域, 因此 草原的碳储蓄能力及其动态变化在全球碳循环中扮 演着重要角色(Scurlock \& Hall, 1998)。我国内蒙古 草原是欧亚草原的一个重要组成部分(Bai et al., 2008), 其经纬度覆盖范围广 $\left(98.0^{\circ}-124.5^{\circ} \mathrm{E}\right.$, $37.7^{\circ}-51.3^{\circ} \mathrm{N}$ ), 导致气温、降雨差异大, 从东到西, 草地类型多样, 分布有温性草甸草原、典型草原和 荒漠草原(马文红, 2006)。不同类型草原的气候因子, 包括气温和降水, 地上、地下生物量(Ma et al., 2008; Yang et al., 2010a)、微生物生物量及组成( Li \& Chen, 2004)均存在显著差异, 这些差异均可能对土壤团 聚体的形成产生影响, 从而导致在不同类型的草原 形成不同的团聚体分布格局。另外, 对于不同的土 层深度, 其湿度、微生物生物量及组成(Allison et al., 2007)、根系生物量(Ma et al., 2008) 以及可溶性有机 碳的淋溶(Sanderman \& Amundson, 2008)等的不同 也可能使团聚体分布存在差异。例如, 在内蒙古半 干早草原耕地向草原过渡的过程中, 由于保护后的 耕地草原与天然草原在地上地下生物量以及土壤质 地等方面有明显差异, 导致了两者在团聚体组分分 配以及稳定性上的差异, 并且这种差异在不同的深 度表现不同(Zhang et al., 2013)。

由于团聚体的形成对土壤有机碳具有物理保护 作用, 土壤团聚体的稳定性在一定程度上决定了土
壤有机碳的稳定性(Bird et al., 2002)。景观尺度的土 壤团聚体稳定性由土壤质地和矿物的变异、土壤有 机质的输入以及干扰共同决定(Lehrsch et al., 1991; Chenu et al., 2000; Abiven et al., 2009)。一项关于草 原生态系统土壤团聚体稳定性多尺度变异的研究发 现, 植被覆盖度越高, 土壤团聚体稳定性越高(Bird et al., 2007)。国内对团聚体稳定性的研究主要集中 在不同的土地利用方式、耕作以及施肥等情况下对 团聚体稳定性的影响(刘恩科等, 2010; 田慎重等, 2013; 李鉴霖等, 2015)。然而, 目前对我国内蒙古草 原土壤团聚体的含量以及稳定性在不同类型以及不 同土层深度的分布规律还不清楚。

考虑到土壤团聚体对于土壤结构和肥力的重要 意义, 以及土壤团聚体稳定性对土壤有机碳的保护 作用, 本研究利用呼伦贝尔-锡林浩特-鄂尔多斯样 带探讨我国内蒙古3种草原类型不同土层深度的土 壤团聚体分布特征及团聚体稳定性分布特征, 具体科 学问题如下: (1)土壤团聚体含量的分布是否因草原 类型和土层深度而异? (2)草原生态系统土壤团聚体 含量的分布与气候因子有何关系? (3)内蒙古3种草原 类型的土壤团聚体稳定性呈现什么样的分布格局?

\section{1 材料和方法}

\section{1 研究区域概况}

本研究区域主要集中在蒙古高原上内蒙古境内 的天然草原。研究样地位于 $107.84^{\circ}-120.06^{\circ} \mathrm{E}$, $39.15^{\circ}-49.62^{\circ} \mathrm{N}$, 海拔564-1 $546 \mathrm{~m}$, 由东北向西南, 内蒙古草原的年平均气温逐渐增加, 年降水量逐渐 减少, 干旱程度逐渐加剧(Wang et al., 2014)。东北半 湿润区、中部半干旱区和西部干旱气候区分别形成 了草甸草原、典型草原和荒漠草原。草甸草原生长 季年降水量 277-327 $\mathrm{mm}$, 生长季平均气温 11.20-13.60 ${ }^{\circ} \mathrm{C}$, 植物种类丰富, 群落以多年生旱 生、中旱生植物占优势。主要建群和优势物种有贝 加尔针茅(Stipa baicalensis)、羊草(Leymus chinensis) 等; 典型草原生长季年降水量174-295 mm, 生长季 年平均气温 $12.60-18.20{ }^{\circ} \mathrm{C}$, 是由典型的旱生性多年 生草本植物组成的群落类型, 是内蒙古草原的主体, 
与草甸草原相比，典型草原的物种丰富程度明显降 低, 群落盖度减小, 主要建群和优势物种有大针茅 (Stipa grandis)、克氏针茅(S. kryovii)等。荒漠草原生 长季年降水量110-219 mm, 生长季年平均气温 14.70-17.60 ${ }^{\circ} \mathrm{C}$, 荒漠草原是由旱生性更强的多年生 矮小草本植物组成的草原群落, 群落生物多样性不 高, 主要有小针茅(S. klemenzii)、短花针茅(S. breviflora)等(马文红, 2006; Ma et al., 2010)(表1)。土壤 类型从东北向西南分别为黑钙土、栗钙土和棕钙土 (Bai et al., 2008)。

气象数据的来源与处理: 对全国670个标准气 象台站1970-2015年间每月平均气温和降水数据进 行克里格插值分析, 然后根据样地的经纬度从插值 结果中提取样地的每月平均气温和降水数据, 以 45 年的平均值作为样地的气温和降水数据。

\section{2 土壤样品采集}

我们于 2015年7-8月沿呼伦贝尔-锡林浩特-鄂 尔多斯一线调查了 3 种草原类型共 78 个样地(图1): 荒漠草原( 20 个样方)、典型草原(45个样方)和草甸草 原(13个样方)。在每处取样点, 设置 $10 \mathrm{~m} \times 10 \mathrm{~m}$ 的 大样方, 沿大样方四角及中心位置分别设置了5个 $1 \mathrm{~m} \times 1 \mathrm{~m}$ 的小样方。之后, 选取大样方其中一条对 角线的 3 个小样方, 并在每个小样方的对角线位置 利用直径 $50 \mathrm{~mm}$ 的土钻采集了至少 3 个剖面的土壤 样品, 采样深度依次为 $0-10 \mathrm{~cm} 、 10-20 \mathrm{~cm} 、 20-30 \mathrm{~cm}$ 、 30-50 cm、50-70 cm 和70-100 cm。采集结束后, 将 小样方内同一土层的土壤样品混合成一个混合样品, 收集后用于室内测定。同时记录样地经纬度、海拔、 坡度和坡向等信息, 每个点的定位信息以及海拔用 GPS测量。

\section{3 土壤样品分析}

所有土壤样品带回实验室风干后, 过 $8 \mathrm{~mm}$ 耖, 以去除根系及石块。我们采用湿篎法进行团聚体分 级(Six et al., 1998), 具体步骤如下: 称取50.00 g风 干土, 将其放置在团聚体分析仪(XY-100, 北京祥宇 伟业仪器设备有限公司, 北京)套篮的最上端篮子 内, 调整桶内水面的高度, 使水面刚好漫过风干土, 将风干土浸泡5 $\mathrm{min}$ 后, 启动仪器进行分级, 以振幅 $3 \mathrm{~cm}$ 振动 $30 \mathrm{~min}$ 后, 根据笁孔径的大小分离出 $<53$ $\mu \mathrm{m} 、 53-250 \mu \mathrm{m} 、 250-2000 \mu \mathrm{m}$ 和 $>2000 \mu \mathrm{m}$ 四级团 聚体组分, 将团聚体套篮小心地从桶内移出, 放置 在实验台上, 然后利用药匙、洗瓶和蒸馏水将各级 笁中的团聚体组分先转移至烧杯中, 再将烧杯中的 才聚体组分和蒸馏水混合物转入相应的铝盒(直径 $60 \mathrm{~mm}$, 高60 mm), 放入烘箱中进行烘干, 烘箱温 度 $50{ }^{\circ} \mathrm{C}$ (不超过 $65{ }^{\circ} \mathrm{C}$ ), 烘干后利用药匙将铝盒中 的团聚体组分转移至称量纸上进行称量; 称量后, 倒回铝盒中, 再加入浓度为 $5 \mathrm{~g} \cdot \mathrm{L}^{-1}$ 的六偏磷酸钠 $\left(\left(\mathrm{NaPO}_{3}\right)_{6}\right)$, 加入的量漫过团聚体1-2 cm, 并放置在 摇床上以中速摇动 $6 \mathrm{~min}$, 以破坏团聚体，过53 $\mu \mathrm{m}$ 笁，使团聚体与砂砾分离，将去除砂砾的团聚体重 新转入铝盒, 烘干并称量。经上述过程后, 得到相应 各级的团聚体及其质量 $m$, 将 $<53 \mu \mathrm{m}$ 、 53-250 $\mu \mathrm{m}$ 、 250-2 $000 \mu \mathrm{m}$ 和 $>2000 \mu \mathrm{m}$ 四级团聚体组分的质量 分别记为 $m 1 、 m 2 、 m 3$ 和 $m 4$ 。

\section{4 数据分析}

\subsection{1 土壤团聚体组分质量百分比的计算}

将团聚体分级后的每级团聚体组分在烘箱中烘 干后称量, 得到每级团聚体组分的质量, 然后将每 级团聚体组分的质量除以用于进行团聚体分级的整

表1 三种草原的基本信息

Table 1 The basic information of the three types of grassland

\begin{tabular}{|c|c|c|c|c|c|}
\hline $\begin{array}{l}\text { 草原类型 } \\
\text { Grassland type }\end{array}$ & $\begin{array}{c}G S P \text { 平均值(范围 })^{1)} \\
\text { Mean of } G S P(\text { range })^{1)}(\mathrm{mm})\end{array}$ & 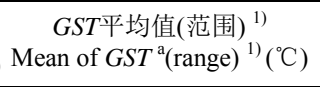 & $\begin{array}{l}\text { 氮:磷平均值(范围) }{ }^{2)} \\
\text { Mean of N:P (range) }\end{array}$ & $\begin{array}{l}\mathrm{pH} \text { 平均值(范围) }{ }^{3)} \\
\text { Mean of } \mathrm{pH}(\text { range })^{3)}\end{array}$ & $\begin{array}{c}\text { 优势种 }^{4)} \\
\text { Dominant species }\end{array}$ \\
\hline $\begin{array}{l}\text { 草甸草原 } \\
\text { Meadow steppe }\end{array}$ & $306(277-327)$ & $12.50(11.20-13.60)$ & $3.83(3.25-4.37)$ & $7.10(7.60-8.00)$ & $\begin{array}{c}\text { 贝加尔针茅 Stipa baicalensis, } \\
\text { 羊草 Leymus chinensis }\end{array}$ \\
\hline $\begin{array}{l}\text { 典型草原 } \\
\text { Typical steppe }\end{array}$ & $242(174-295)$ & $14.00(12.60-18.20)$ & $3.02(2.93-3.11)$ & $7.50(7.26-7.73)$ & $\begin{array}{l}\text { 大针茅 Stipa grandis, } \\
\text { 克氏针茅 Stipa kryovii }\end{array}$ \\
\hline $\begin{array}{l}\text { 荒漠草原 } \\
\text { Desert steppe }\end{array}$ & $163(110-219)$ & $16.50(14.70-17.60)$ & $2.48(2.41-2.54)$ & $7.80(6.65-7.56)$ & $\begin{array}{l}\text { 小针茅 Stipa klemenzii, } \\
\text { 短花针茅 Stipa breviflora }\end{array}$ \\
\hline
\end{tabular}

GSP, 生长季降水量; GST, 生长季年平均气温。1), 数据来自 Ma等(2010); 2), 数据来自Yang等(2014); 3), 数据来自Yang等(2012); 4), 数据来自马文红 (2006)。

GSP, growing-season precipitation; GST, mean growing-season air temperature. 1), data are from Ma et al., 2010; 2), data are from Yang et al., 2014; 3), data are from Yang et al., 2012; 4), data are from Ma, 2006. 


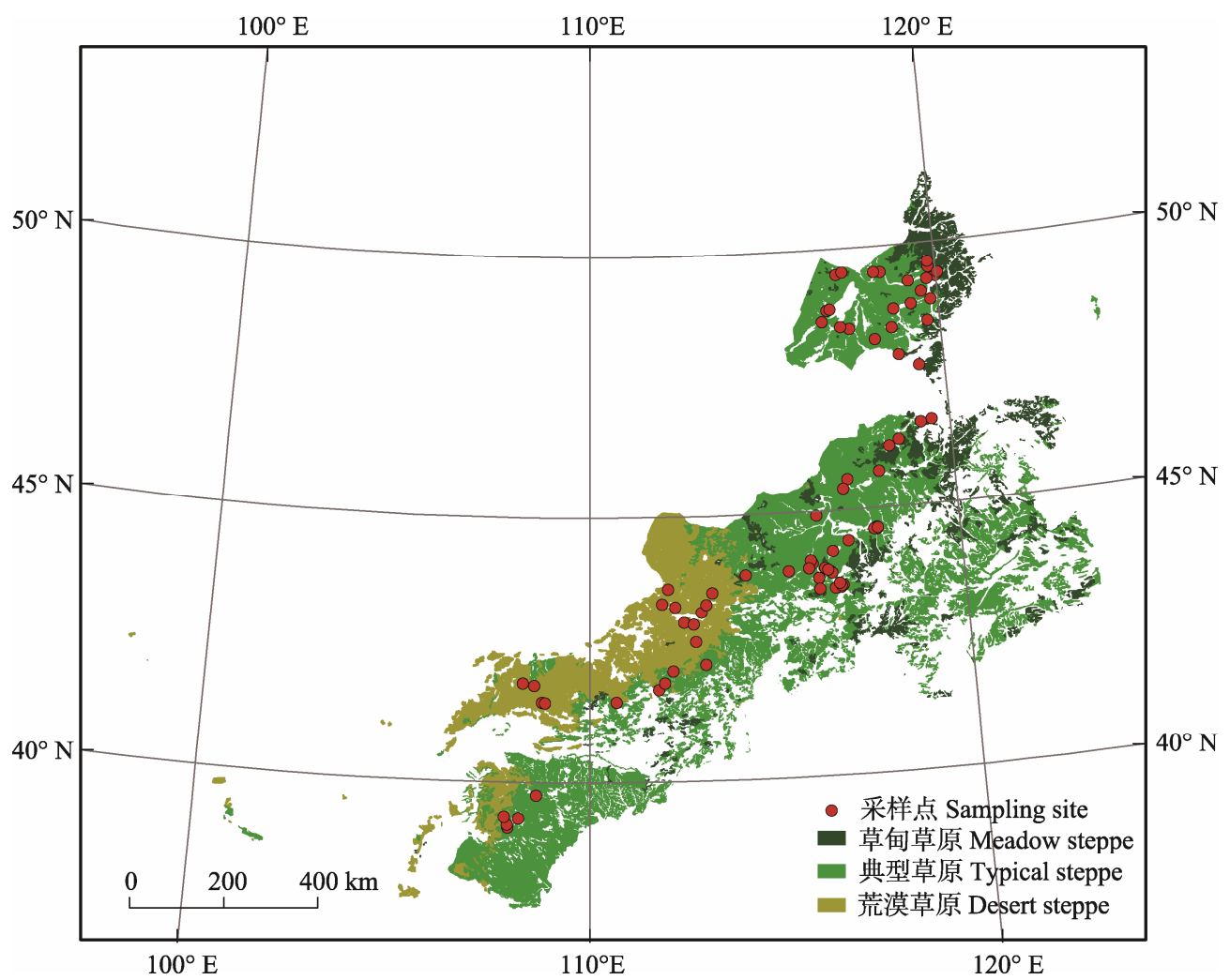

图1 采样点分布图。

Fig. 1 The distribution of sampling sites.

土的质量, 在我们的研究中指 $50 \mathrm{~g}$ 风干土质量, 得 到每级团聚体组分的质量百分比(\%), 分别记为 $m p 1$ (<53 $\mu \mathrm{m}$ 团聚体组分的质量百分比)、 $m p 2$ (53$250 \mu \mathrm{m}$ 团聚体组分的质量百分比)、mp3 (250-2 000 $\mu \mathrm{m}$ 团聚体组分的质量百分比)和 $m p 4$ (> $2000 \mu \mathrm{m}$ 团 聚体组分的质量百分比), 具体公式如下:

$$
m p(i)=\sum \frac{m(i)}{M} \times 100 \%
$$

式中: $m p(i)$ 指每级团聚体组分的质量百分比; $m(i)$ 指 每级团聚体组分的质量; $i$ 是各级团聚体组分的编号 (1-4分别指 $<53 \mu \mathrm{m} 、 53-250 \mu \mathrm{m} 、 250-2000 \mu \mathrm{m}$ 和 $>2000 \mu \mathrm{m}$ 团聚体组分); $M$ 指用于进行团聚体分级 的整土质量, 在我们的研究中 $M=50 \mathrm{~g}$ 。

\subsection{2 土壤团聚体稳定性的计算}

到目前为止，测定土壤团聚体稳定性的方法有 很多, 包括了水稳性团聚体质量占比、团聚体形成 指数、团聚体破坏指数、标准化稳定性指数、稳定 性团聚体和稳定性大团聚体指数、平均质量直径 $(M M D)$ 和几何平均直径 $(G M D)$ 等(Marquez et al., 2004)。其中, $M M D$ 是表征团聚体稳定性的最重要、 最常用的指标(Nimmo \& Perkins, 2002), 是土壤团
聚体组分分布的综合参数, 从而作为表征整土结构 的指数(Amezketa, 1999)。GMD同样也能作为团聚体 大小分布的一种指数。 $M M D$ 与 $G M D$ 作为反映土壤 团聚体状况的指标, 其值越大表示土壤的团聚度越 高, 团聚体稳定性越强(Nimmo \& Perkins, 2002)。其 计算方法如下:

$$
\begin{aligned}
M M D & =\Sigma n X_{i} M_{i} \\
G M D & =\exp \left[\Sigma n M_{i} \ln X_{i}\right]
\end{aligned}
$$

式中: $n$ 为粒径分组的组数; $X_{i}$ 为这一粒径组分的平 均直径, $i$ 是各级团聚体组分的编号(1-4 分别指 $<53 \mu \mathrm{m} 、 53-250 \mu \mathrm{m} 、 250-2000 \mu \mathrm{m}$ 和 $>2000 \mu \mathrm{m}$ 团聚体组分) ( $X_{1}=26.5 \mu \mathrm{m} ; X_{2}=151.5 \mu \mathrm{m} ; X_{3}=$ $\left.1125 \mu \mathrm{m} 、 X_{4}=2000 \mu \mathrm{m}\right) ; M_{i}$ 为这一粒径团聚体的 质量分数。

\subsection{3 统计分析}

利用双因素方差分析的方法分析草原类型和土 层深度对各层土壤团聚体质量百分比和团聚体稳定 性分布的影响。利用一元线性回归分析土壤团聚体 质量百分比及团聚体稳定性与年降水量和年平均气 温的关系。利用多重比较分析不同草地类型, 及不 同土层深度的土壤团聚体质量百分比间的分布差异 
以及土壤团聚体稳定性的差异。所有的统计分析均 由R(3.3.1)软件(R Core Team (2017))完成。柱状图在 SigmaPlot 12.0软件中绘制。本研究中空间制图在地 理信息系统软件ArcGIS 10.0中完成。

\section{2 结果和分析}

\section{1 土壤团聚体质量百分比的分配特征}

总体而言，草甸草原、典型草原及荒漠草原在 0-1 m中的总团聚体质量百分数分别为 $(49.52 \pm$ $2.76) \% 、(32.32 \pm 1.08) \%$ 和 $(25.22 \pm 1.17) \%$ (表 2$)$; 具 体每一层中每一级的团聚体质量百分比分布见附录 I。双因素方差分析结果显示: 土层深度对团聚体分 配效应不显著 $(p=0.12)$, 且两者的交互效应也不显 著 $(p=0.41)$, 但草原类型的效应显著 $(p<0.01)$ (附 录II)。对3种草原在不同土层深度上的总团聚体质量 百分数进行统计分析, 结果表明: 在每一层土层, 草甸草原都显著大于典型草原与荒漠草原 $(p<0.01)$, 而典型草原与荒漠草原之间无显著差异 $(p>0.05)$ (表2)。另外, 3种类型草原的土壤团聚体含量在各个 土层深度都没有明显的规律(表2)。

\section{2 土壤团聚体含量的分布与气候因子的关系}

进一步分析草原生态系统土壤团聚体含量的分
布与气候因子之间的关系。结果(表3)表明: 在深度 小于 $50 \mathrm{~cm}$ 的土层中, 总团聚体的质量百分比与年 降水量 $(M A P)$ 显著正相关 $(p<0.01)$, 而在大于 $50 \mathrm{~cm}$ 的土层中，总团聚体的质量百分比与年降水量几近 正相关关系 $(p=0.06)($ 表 3$)$ 。

土层深度在 $70 \mathrm{~cm}$ 以上的总团聚体的质量百分 比与年平均气温呈显著负相关关系 $(p<0.01)$, 而 70-100 cm土层 $(p=0.11)$ 的总团聚体的质量百分比 与年平均气温 $(M A T)$ 无显著负相关关系 $($ 表 3$)$ 。

\section{3 土壤团聚体稳定性的分配特征}

草甸草原与典型草原土壤团聚体的 $M M D$ 与 $G M D$ 随着土层深度均呈逐渐降低的趋势, 而荒漠 草原并无明显的规律(图2), 具体结果见附录III。统 计分析结果表明: 在0-10 $\mathrm{cm}$ 和10-20 cm土层中, 草 甸草原土壤团聚体的 $M M D$ 与 $G M D$ 都显著高于典型 草原和荒漠草原 $(p<0.05)$, 但在其他土层 3 种草原 土壤团聚体的 $M M D$ 与 $G M D$ 都没有显著差异(图2)。 对于草甸草原, 0-10 $\mathrm{cm}$ 土壤团聚体的 $M M D$ 与 $G M D$ 都显著高于其他土层 $(p<0.05)$; 对于典型草原，小 于30 $\mathrm{cm}$ 土层土壤团聚体的 $M M D$ 与 $G M D$ 都显著高 于大于 $30 \mathrm{~cm}$; 对于荒漠草原, 各层土壤团聚体的 $M M D$ 与 $G M D$ 都无显著差异(图2)。

表2 三种草原各层土壤团聚体的质量百分比(平均值土标准误差)

Table 2 The mass percentage (\%) of total soil aggregate in different soil layers (mean $\pm S E$ )

\begin{tabular}{lccc}
\hline 土层 Soil layer $(\mathrm{cm})$ & 草甸草原 Meadow steppe $(\%)$ & 典型草原 Typical steppe $(\%)$ & 荒漠草原 Desert steppe $(\%)$ \\
\hline $0-10$ & $49.66 \pm 1.12^{\mathrm{a}}$ & $35.07 \pm 2.20^{\mathrm{b}}$ & $27.25 \pm 1.87^{\mathrm{b}}$ \\
$10-20$ & $48.63 \pm 2.08^{\mathrm{a}}$ & $32.30 \pm 2.19^{\mathrm{b}}$ & $26.84 \pm 2.76^{\mathrm{b}}$ \\
$20-30$ & $52.15 \pm 5.70^{\mathrm{a}}$ & $31.80 \pm 2.32^{\mathrm{b}}$ & $24.52 \pm 2.68^{\mathrm{b}}$ \\
$30-50$ & $48.20 \pm 1.79^{\mathrm{a}}$ & $31.53 \pm 2.28^{\mathrm{b}}$ & $24.45 \pm 3.26^{\mathrm{b}}$ \\
$50-70$ & $49.14 \pm 14.21^{\mathrm{a}}$ & $31.21 \pm 0.82^{\mathrm{b}}$ & $21.04 \pm 2.49^{\mathrm{b}}$ \\
$70-100$ & $48.40 \pm 15.21^{\mathrm{a}}$ & $28.82 \pm 1.5^{\mathrm{b}}$ & $23.10 \pm 3.73^{\mathrm{b}}$ \\
$0-100$ & $49.52 \pm 2.76^{\mathrm{a}}$ & $32.32 \pm 1.08^{\mathrm{b}}$ & $25.22 \pm 1.17^{\mathrm{b}}$ \\
\hline
\end{tabular}

小写字母表示在同一层中 3 种草原类型在 $5 \%$ 水平上的差异。

Lowercase letters represent the differences of aggregate fraction in the same soil layer among three types of grassland at the level of $5 \%$.

表3 各层土壤团聚体总质量百分数与气候因子的一元线性关系

Table 3 The linear relationships between total aggregate mass percentage of different soil layers and climate factors

\begin{tabular}{|c|c|c|c|c|c|c|c|c|}
\hline \multirow{2}{*}{$\begin{array}{l}\text { 土层 } \\
\text { Soil layer }(\mathrm{cm})\end{array}$} & \multicolumn{4}{|c|}{ 年降水量 Mean annual precipitation (MAP) (mm) } & \multicolumn{4}{|c|}{ 年平均气温 Mean annual air temperature $(M A T)\left({ }^{\circ} \mathrm{C}\right)$} \\
\hline & $a$ & $b$ & $R^{2}$ & $p$ & $a$ & $b$ & $R^{2}$ & $p$ \\
\hline $0-10$ & 0.13 & -2.10 & 0.26 & $<0.01$ & -2.78 & 41.06 & 0.20 & $<0.01$ \\
\hline $10-20$ & 0.12 & 0.17 & 0.18 & $<0.01$ & -2.59 & 39.65 & 0.14 & $<0.01$ \\
\hline $20-30$ & 0.13 & -4.05 & 0.20 & $<0.01$ & -3.21 & 10.24 & 0.20 & $<0.01$ \\
\hline $30-50$ & 0.12 & -1.64 & 0.16 & $<0.01$ & -3.21 & 39.26 & 0.21 & $<0.01$ \\
\hline 50-70 & 0.13 & -7.19 & 0.11 & 0.06 & -3.32 & 38.21 & 0.15 & 0.03 \\
\hline $70-100$ & 0.14 & -11.35 & 0.11 & 0.06 & -2.73 & 36.29 & 0.07 & 0.11 \\
\hline
\end{tabular}

当 $p<0.05$ 时, 一元线性关系显著; $Y=a M A P+b ; Y=a M A T+b, Y$ 表示土壤团聚体总质量百分数, $a$ 是系数, $b$ 是常数。

The linear relationship is significant when $p<0.05 ; Y=a M A P+b ; Y=a M A T+b$. Where $Y$ is the total mass percentage of soil aggregate, $a$ is a coefficient, $b$ is a constant.

www.plant-ecology.com 


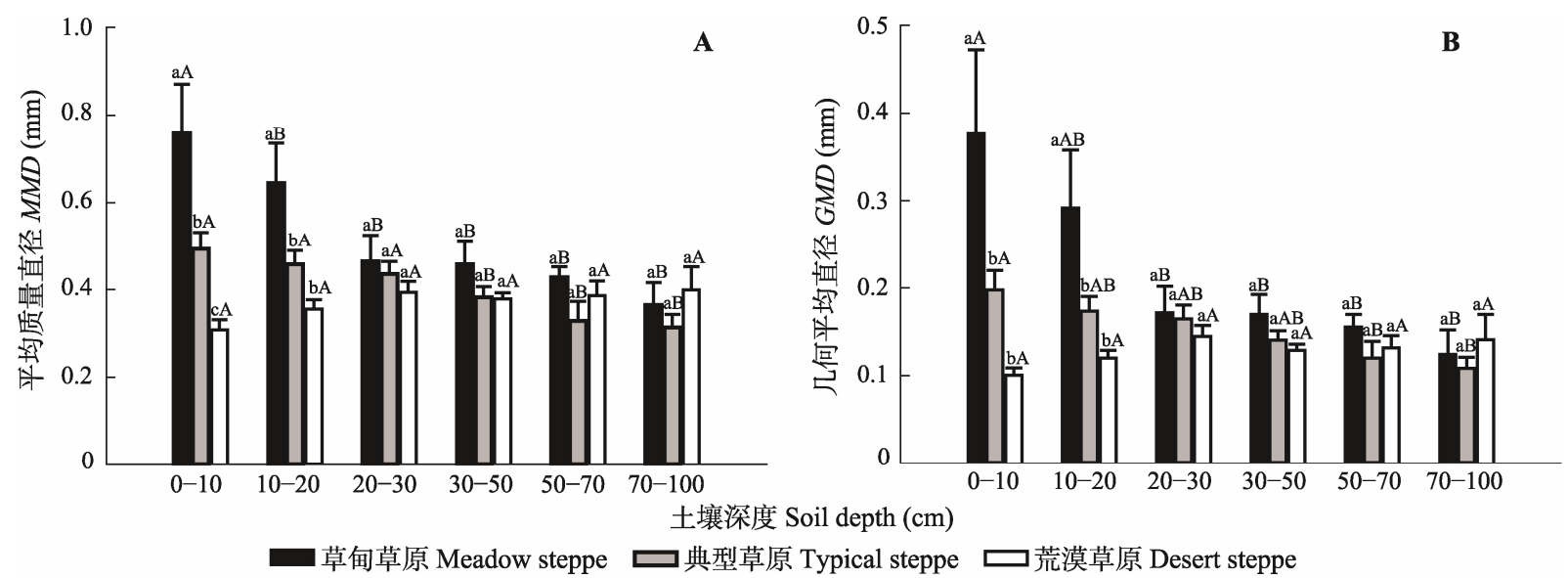

图2 三种草原类型在不同土壤深度上的平均质量直径(A)和几何平均直径(B) (平均值标准误差)。小写字母表示在同一土层 中3种草原类型在 $5 \%$ 水平上的差异; 大写字母表示同一种草原类型不同土壤深度在 $5 \%$ 水平上的差异。

Fig. 2 Mean mass diameter (MMD) (A) and geometric mean diameter (GMD) (B) of soil aggregates at different soil depths in three types of grassland (mean $\pm S E$ ). Lowercase letters represent the differences of $M M D$ and $G M D$ in the same soil layer among three types of grassland at the level of 5\%. Uppercase letters represent the differences of MMD and GMD among different soil layers in one type of grassland at the level of $5 \%$.

\section{3 讨论}

土壤团聚体是土壤有机碳的重要物理保护方式, 其稳定性与土壤有机碳稳定性密切相关(Bird et al., 2002; Denef et al., 2007)。因此，探讨土壤团聚体形 成及稳定的分布格局对于理解生态系统土壤健康及 有机碳库稳定性具有重要意义。本研究分析了我国 内蒙古不同草原类型土壤团聚体在不同气候梯度及 土层深度的分布特征, 发现草原类型对土壤团聚体 的形成和稳定具有显著影响, 且土壤团聚体含量随 年降水量和年平均气温呈现一定的分布格局。

\section{1 土壤团聚体的质量分布}

土壤团聚体是通过结合体(如细根、微生物菌 丝、糖分及黏粒、粉粒等)将土壤颗粒黏结在一起而 形成的(Six et al., 2002b), 因此影响结合体的形式和 量的因素均可能影响土壤团聚体的形成和分布。有 研究发现, 内蒙古草原大部分根系主要分布在0$30 \mathrm{~cm}$ 土层(Abrishamkesh et al., 2011); 微生物生物 量碳的分布随着土层深度的增加而减少(Blume et al., 2002)。因此, 我们期待团聚体质量百分数随土 层深度增加而减少, 而本文的结果显示土层深度对 土壤总团聚体的质量百分数并无显著影响。这可能 是因为形成大团聚体的有机质较多来源于调落物、 根系和微生物等, 而形成小团聚体的有机质来源并 不确定, 导致了大团聚体的含量随着土层深度逐渐 减少而小团聚体的含量上升(附录I), 因而总团聚体 质量百分数不一定受土层深度的影响。另外, 我们
发现草原类型对总团聚体组分的影响显著，表明内 蒙古不同草原类型的土壤团聚体分布具有显著差异, 这可能与不同草原类型的气候因子(包括气温和降 雨, 地上、地下生物量(Ma et al., 2008)以及微生物生 物量及组成(Li \& Chen, 2004))密切相关。在一项关 于内蒙古半干旱草原退耕还草的研究中, 在0$30 \mathrm{~cm}$ 的土层内, 尽管退耕还草的样地地上和地下 生物量随着保护时间推移而增加, 但在研究期间, 天然草原的地上和地下生物量始终都显著高于退耕 还草的样地, 导致天然草地在0-30 $\mathrm{cm}$ 土层中的团 聚体含量以及团聚体的稳定性都显著高于退耕还草 的样地(Zhang et al., 2013), 本研究与之相似。

\section{2 土壤团聚体含量的分布与气候因子的关系}

降水和气温是影响各个生态系统过程的重要因 子, 包括物种分布, 生态系统碳、水及养分循环等 (Luo et al., 2008; Austin \& van Niel, 2011; Wu et al., 2011)。例如，中国草原地上、地下生物量及土壤有 机碳库的分布格局均受年降水量及年平均气温的影 响(Yang et al., 2010a, 2010b), 而内蒙古草原土壤团 聚体含量的分布格局与降水和气温的关系并不清 楚。本样带调查的结果表明, 总团聚体的质量百分 数与年降水量呈正相关关系, 而与年平均气温呈负 相关关系(表2), 表明在内蒙古草原生态系统, 降水 量高能促进土壤团聚体的形成, 气温高则不利于土 壤团聚体的形成。这可能是因为降水量高, 导致地 上、地下生物量也高(Yang et al., 2010a), 使得有机 碳输入量高, 而促进了土壤团聚体的形成。另外, 
$M A T$ 与 $M A P$ 对深层 $(50-70 \mathrm{~cm} 、 70-100 \mathrm{~cm})$ 土壤的团 聚体含量的分布并无显著影响, 这可能是因为气候 主要通过影响植物碳输入而影响土壤团聚体的形成, 而深层土壤受植物碳输入的影响较小, 更受土壤质 地的影响(Jobbágy \& Jackson, 2000)。

\section{3 土壤团聚体稳定性与草原类型及土层深度的} 关系

土壤团聚体的 $M M D$ 和 $G M D$ 是反映土壤团聚体 稳定性的重要指标(Kemper \& Chepil, 1965)。本研究 结果表明, $M M D$ 与 $G M D$ 在不同草原类型以及不同 土壤深度的趋势一致。 $20 \mathrm{~cm}$ 以上的土层深度中, 草 甸草原土壤团聚体的 $M M D$ 和 $G M D$ 均显著高于典型 和荒漠草原土壤团聚体的 $M M D$ 和 $G M D$, 这可能是 因为草甸草原较典型草原和荒漠草原湿润, 拥有的 物种多样性丰富, 草群盖度较高(李博, 1979), 从而 导致根系发达, 而根系对土壤团聚体的形成和稳定 性有很大影响(Pinheiro et al., 2004)。另有研究结果 表明, 随着土层深度增加, $M M D$ 和 $G M D$ 都呈现降低 的趋势(赵世伟等, 2006; 陈帅和孙涛, 2017), 我们 的研究结果与之相似。本研究也发现草甸草原和典 型草原土壤团聚体的 $M M D$ 和 $G M D$ 随着土层深度逐 渐增加而降低, 这可能是因为湿度、微生物生物量 及组成随着土层深度逐渐增加而降低, 从而导致形 成团聚体的黏合剂含量降低, 进一步导致了团聚体 稳定性在深度上的变化(Allison et al., 2007)。然而, 荒漠草原土壤团聚体的 $M M D$ 和 $G M D$ 并没有明确的 规律, 这可能是因为荒漠草原植被覆盖度低, 其土 壤微生物生物量及土壤有机碳含量也低, 使得土壤 团聚体稳定性低(Bird et al., 2007), 从而导致在土层 深度上差异不明显。

\section{基金项目 国家重点基础研究发展计划(973计划) (2015CB954201和2014CB954303)。}

\section{参考文献}

Abiven S, Menasseri S, Chenu C (2009). The effects of organic inputs over time on soil aggregate stability-A literature analysis. Soil Biology \& Biochemistry, 41, 1-12.

Abrishamkesh S, Gorji M, Asadi H (2011). Long-term effects of land use on soil aggregate stability. International Agrophysics, 25, 103-108.

Allison V, Yermakov Z, Miller R, Jastrow J, Matamala R (2007). Using landscape and depth gradients to decouple the impact of correlated environmental variables on soil microbial community composition. Soil Biology \& Bio- chemistry, 39, 505-516.

Amezketa E (1999). Soil aggregate stability: A review. Journal of Sustainable Agriculture, 14, 83-151.

Austin MP, van Niel KP (2011). Improving species distribution models for climate change studies: Variable selection and scale. Journal of Biogeography, 38, 1-8.

Bai YF, Wu JG, Xing Q, Pan QM, Huang JH, Yang DL, Han XG (2008). Primary production and rain use efficiency across a precipitation gradient on the Mongolia Plateau. Ecology, 89, 2140-2153.

Bird SB, Herrick JE, Wander M, Wright S (2002). Spatial heterogeneity of aggregate stability and soil carbon in semiarid rangeland. Environmental Pollution, 116, 445-455.

Bird SB, Herrick JE, Wander MM, Murray L (2007). Multi-scale variability in soil aggregate stability: Implications for understanding and predicting semi-arid grassland degradation. Geoderma, 140, 106-118.

Blume E, Bischoff M, Reichert J, Moorman T, Konopka A, Turco R (2002). Surface and subsurface microbial biomass, community structure and metabolic activity as a function of soil depth and season. Applied Soil Ecology, 20, 171-181.

Cavagnaro T, Jackson L, Six J, Ferris H, Goyal S, Asami D, Scow K (2006). Arbuscular mycorrhizas, microbial communities, nutrient availability, and soil aggregates in organic tomato production. Plant and Soil, 282, 209-225.

Chen S, Sun T (2017). Research of soil aggregate stability in different degradation stages of Songnen grassland. Pratacultural Science, 34, 217-223. (in Chinese with English abstract) [陈帅, 孙涛 (2017). 松嫩草地不同退化阶段 的土壤团聚体稳定性. 草业科学, 34, 217-223.]

Chenu C, Le Bissonnais Y, Arrouays D (2000). Organic matter influence on clay wettability and soil aggregate stability. Soil Science Society of America Journal, 64, 1479-1486.

Denef K, Zotarelli L, Boddey RM, Six J (2007). Microaggregate-associated carbon as a diagnostic fraction for management-induced changes in soil organic carbon in two oxisols. Soil Biology \& Biochemistry, 39, 1165-1172.

Jastrow JD (1996). Soil aggregate formation and the accrual of particulate and mineral-associated organic matter. Soil Biology \& Biochemistry, 28, 665-676.

Jobbágy EG, Jackson RB (2000). The vertical distribution of soil organic carbon and its relation to climate and vegetation. Ecological Applications, 10, 423-436.

Kemper W, Chepil W (1965). Size distribution of aggregates. In: Black CA ed. Physical and Mineralogical Properties. American Society of Agronomy, Madison. 499-510.

Lal R (2000). Physical management of soils of the tropics: Priorities for the 21st Century. Soil Science, 165, 191-207.

Lehrsch G, Sojka R, Carter D, Jolley P (1991). Freezing effects on aggregate stability affected by texture, mineralogy, and organic matter. Soil Science Society of America Journal, 55, 1401-1406.

Li B (1979). The general characteristics of grassland vegetation in China. Journal of Chinese Grassland, 1, 2-12. [李博 (1979). 中国草原植被的一般特征. 中国草地学报, 1,

www.plant-ecology.com 
2-12.]

Li JL, Jiang CS, Hao QJ (2015). Distribution characteristics of soil organic carbon and its physical fractions under the different land uses in Jinyun Mountain. Acta Ecologica Sinica, 35, 3733-3742. (in Chinese with English abstract) [李鉴霖, 江长胜, 郝庆菊 (2015). 缙云山不同土地利 用方式土壤有机碳组分特征。生态学报, 35 , 3733-3742.]

Li XZ, Chen ZZ (2004). Soil microbial biomass C and N along a climatic transect in the Mongolian steppe. Biology and Fertility of Soils, 39, 344-351.

Liu EK, Zhao BQ, Mei XR, Li XY, Li J (2010). Distribution of water-stable aggregates and organic carbon of arable soils affected by different fertilizer application. Acta Ecologica Sinica, 30, 1035-1041. (in Chinese with English abstract) [刘恩科, 赵秉强, 梅旭荣, 李秀英, 李娟 (2010). 不同 施肥处理对土壤水稳定性团聚体及有机碳分布的影响. 生态学报, 30, 1035-1041.]

Luo YQ, Gerten D, Le Maire G, Parton WJ, Weng ES, Zhou XH, Keough C, Beier C, Ciais P, Cramer W (2008). Modeled interactive effects of precipitation, temperature, and $\left[\mathrm{CO}_{2}\right]$ on ecosystem carbon and water dynamics in different climatic zones. Global Change Biology, 14, 1986-1999.

Ma WH (2006). Carbon Storage in Temperate Grassland of Inner Mongolia. $\mathrm{PhD}$ dissertation. Peking University, Beijing. 18-19. (in Chinese with English abstract) [马文红 (2006). 内蒙古温带草地的碳储量. 博士学位论文. 北 京大学, 北京. 18-19.]

Ma WH, He JS, Yang YH, Wang XP, Liang CZ, Anwar M, Zeng H, Fang JY, Schmid B (2010). Environmental factors covary with plant diversity-productivity relationships among Chinese grassland sites. Global Ecology and Biogeography, 19, 233-243.

Ma WH, Yang YH, He JS, Zeng H, Fang JY (2008). Aboveand belowground biomass in relation to environmental factors in temperate grasslands, Inner Mongolia. Science China Series C: Life Sciences, 51, 263-270.

Marquez CO, Garcia VJ, Cambardella CA, Schultz RC, Isenhart TM (2004). Aggregate-size stability distribution and soil stability. Soil Science Society of America Journal, 68, 725-735.

Nimmo JR, Perkins KS (2002). Aggregate stability and size distribution. In: Dane JH, Topp GC eds. Methods of Soil Analysis. American Society of Agronomy, Madison. 317-328.

Pinheiro E, Pereira M, Anjos L (2004). Aggregate distribution and soil organic matter under different tillage systems for vegetable crops in a red latosol from brazil. Soil and Tillage Research, 77, 79-84.

Sanderman J, Amundson R (2008). A comparative study of dissolved organic carbon transport and stabilization in california forest and grassland soils. Biogeochemistry, 89, 309-327.

Scurlock J, Hall D (1998). The global carbon sink: A grassland perspective. Global Change Biology, 4, 229-233.

Six J, Conant R, Paul EA, Paustian K (2002a). Stabilization mechanisms of soil organic matter: Implications for C-saturation of soils. Plant and Soil, 241, 155-176.

Six J, Elliott E, Paustian K, Doran JW (1998). Aggregation and soil organic matter accumulation in cultivated and native grassland soils. Soil Science Society of America Journal, 62, 1367-1377.

Six J, Feller C, Denef K, Ogle SM, de Moraes Sa JC, Albrecht A (2002b). Soil organic matter, biota and aggregation in temperate and tropical soils-effects of no-tillage. Agronomie, 22, 755-775.

Tian SZ, Wang Y, Li N, Ning TY, Wang BW, Zhao HX, Li ZJ (2013). Effects of different tillage and straw systems on soil water-stable aggregate distribution and stability in the North China Plain. Acta Ecologica Sinica, 33, 7116-7124. (in Chinese with English abstract) [田慎重, 王瑜, 李娜, 宁堂原, 王丙文, 赵红香, 李增嘉 (2013). 耕作方式和 秸秆还田对华北地区农田土壤水稳性团聚体分布及稳 定性的影响. 生态学报, 33, 7116-7124.]

Wang C, Wang XB, Liu DW, Wu HH, Lü XT, Fang YT, Cheng WX, Luo WT, Jiang P, Shi J (2014). Aridity threshold in controlling ecosystem nitrogen cycling in arid and semi-arid grasslands. Nature Communications, 5, 4799.

Wu ZT, Dijkstra P, Koch GW, Penuelas J, Hungate BA (2011). Responses of terrestrial ecosystems to temperature and precipitation change: A meta-analysis of experimental manipulation. Global Change Biology, 17, 927-942.

Yang YH, Fang JY, Ji CJ, Datta A, Li P, Ma WH, Mohammat A, Shen HH, Hu HF, Knapp BO, Smith P (2014). Stoichiometric shifts in surface soils over broad geographical scales: Evidence from China's grasslands. Global Ecology and Biogeography, 23, 947-955.

Yang YH, Fang JY, Ma WH, Guo D, Mohammat A (2010a). Large-scale pattern of biomass partitioning across China's grasslands. Global Ecology and Biogeography, 19, 268-277.

Yang YH, Fang JY, Ma WH, Smith P, Mohammat A, Wang SP, Wang W (2010b). Soil carbon stock and its changes in northern China's grasslands from 1980s to 2000s. Global Change Biology, 16, 3036-3047.

Yang YH, Ji CJ, Ma WH, Wang SF, Wang SP, Han WX, Mohammat A, Robinson D, Smith P (2012). Significant soil acidification across northern china's grasslands during 1980s-2000s. Global Change Biology, 18, 2292-2300.

Zhang ZH, Li XY, Jiang ZY, Peng HY, Li L, Zhao GQ (2013). Changes in some soil properties induced by re-conversion of cropland into grassland in the semiarid steppe zone of Inner Mongolia, China. Plant and Soil, 373, 89-106.

Zhao SW, Su J, Wu JS, Yang YH, Liu NN (2006). Changes of soil aggregate organic carbon during process of vegetation restoration in Ziwuling. Journal of Soil and Water Conservation, 20, 114-117. (in Chinese with English abstract) [赵世伟, 苏静, 吴金水, 杨永辉, 刘娜娜 (2006). 子午 岭植被恢复过程中土壤团聚体有机碳含量的变化. 水 土保持学报, 20, 114-117.]

责任编委: 程晓莉 责任编辑: 王 蒇 
附录I 各个土层各级团聚体的质量百分比(平均值土标准误差)

Appendix I The mass percentage (\%) of each aggregate fraction in different soil layers (mean $\pm S E$ )

\begin{tabular}{|c|c|c|c|c|}
\hline \multicolumn{2}{|c|}{ 土层 Soil layer (cm) } & \multirow{2}{*}{$\begin{array}{c}\text { 草甸草原 Meadow steppe (\%) } \\
14.15 \pm 1.98^{\mathrm{a}}\end{array}$} & \multirow{2}{*}{$\begin{array}{c}\text { 典型草原 } \\
\text { Typical steppe (\%) } \\
13.58 \pm 1.20^{\mathrm{a}}\end{array}$} & \multirow{2}{*}{$\begin{array}{c}\text { 荒漠草原 Desert steppe (\%) } \\
14.8 \pm 1.49^{\mathrm{a}}\end{array}$} \\
\hline $0-10$ & $\mathrm{~A} 1$ & & & \\
\hline & A2 & $9.33 \pm 0.93^{\mathrm{a}}$ & $8.32 \pm 0.44^{\mathrm{a}}$ & $5.90 \pm 0.45^{\mathrm{a}}$ \\
\hline & $\mathrm{A} 3$ & $15.90 \pm 2.96^{\mathrm{a}}$ & $10.33 \pm 1.24^{\mathrm{ab}}$ & $6.76 \pm 0.71^{\mathrm{b}}$ \\
\hline & A4 & $14.15 \pm 4.48^{\mathrm{a}}$ & $5.93 \pm 0.99^{\mathrm{b}}$ & - \\
\hline \multirow[t]{3}{*}{$10-20$} & $\mathrm{~A} 1$ & $18.82 \pm 3.41^{\mathrm{a}}$ & $12.89 \pm 1.02^{\mathrm{ab}}$ & $12.67 \pm 1.30^{\mathrm{b}}$ \\
\hline & $\mathrm{A} 2$ & $10.37 \pm 1.30^{\mathrm{a}}$ & $8.30 \pm 0.59^{\mathrm{a}}$ & $6.84 \pm 1.09^{\mathrm{a}}$ \\
\hline & $\mathrm{A} 3$ & $15.43 \pm 3.08^{\mathrm{a}}$ & $9.30 \pm 0.93 \mathrm{a}^{\mathrm{b}}$ & $7.32 \pm 0.90^{\mathrm{b}}$ \\
\hline \multirow[t]{5}{*}{$20-30$} & A4 & $10.22 \pm 2.87^{\mathrm{a}}$ & $4.51 \pm 0.96^{\mathrm{b}}$ & - \\
\hline & A1 & $22.86 \pm 3.23^{\mathrm{a}}$ & $13.70 \pm 1.39^{\mathrm{ab}}$ & $10.14 \pm 1.16^{\mathrm{b}}$ \\
\hline & A2 & $12.93 \pm 1.34^{\mathrm{a}}$ & $7.65 \pm 0.52^{\mathrm{ab}}$ & $6.64 \pm 0.65^{\mathrm{b}}$ \\
\hline & A3 & $12.39 \pm 2.54^{\mathrm{a}}$ & $9.24 \pm 0.95^{\mathrm{a}}$ & $7.74 \pm 1.35^{\mathrm{a}}$ \\
\hline & A4 & $6.95 \pm 1.35^{\mathrm{a}}$ & $3.72 \pm 1.03^{\mathrm{a}}$ & - \\
\hline \multirow[t]{4}{*}{$30-50$} & $\mathrm{~A} 1$ & $18.97 \pm 2.43^{\mathrm{a}}$ & $13.55 \pm 1.16^{\mathrm{ab}}$ & $10.30 \pm 1.20^{\mathrm{b}}$ \\
\hline & A2 & $14.70 \pm 2.61^{\mathrm{a}}$ & $8.74 \pm 0.82^{\mathrm{ab}}$ & $6.93 \pm 1.53^{\mathrm{b}}$ \\
\hline & A3 & $13.39 \pm 2.83^{\mathrm{a}}$ & $8.02 \pm 0.75^{\mathrm{ab}}$ & $6.75 \pm 0.66^{b}$ \\
\hline & A4 & $4.76 \pm 0.79^{\mathrm{a}}$ & $3.70 \pm 1.17^{\mathrm{a}}$ & - \\
\hline \multirow[t]{4}{*}{$50-70$} & A1 & $23.89 \pm 8.50^{\mathrm{a}}$ & $17.02 \pm 3.26^{\mathrm{b}}$ & $9.43 \pm 1.50^{\mathrm{c}}$ \\
\hline & $\mathrm{A} 2$ & $12.34 \pm 3.64^{\mathrm{a}}$ & $7.18 \pm 1.09^{\mathrm{ab}}$ & $5.32 \pm 0.68^{b}$ \\
\hline & A3 & $17.35 \pm 5.94^{\mathrm{a}}$ & $6.84 \pm 1.28^{\mathrm{b}}$ & $6.29 \pm 0.83^{b}$ \\
\hline & A4 & - & - & - \\
\hline \multirow[t]{4}{*}{$70-100$} & A1 & $29.72 \pm 9.86^{\mathrm{a}}$ & $14.71 \pm 3.01^{\mathrm{b}}$ & $10.09 \pm 1.22^{\mathrm{b}}$ \\
\hline & A2 & $10.79 \pm 2.97^{\mathrm{a}}$ & $6.87 \pm 1.08^{\mathrm{a}}$ & $5.15 \pm 1.150^{\mathrm{a}}$ \\
\hline & A3 & $14.68 \pm 5.49^{\mathrm{a}}$ & $6.65 \pm 1.46^{\mathrm{b}}$ & $7.86 \pm 2.23^{\mathrm{b}}$ \\
\hline & A4 & - & - & - \\
\hline
\end{tabular}

小写字母表示在同一土层中 3 种草原类型在 $5 \%$ 水平上的差异。 $\mathrm{A} 1,<53 \mu \mathrm{m}$ 的团聚体; $\mathrm{A} 2,53-250 \mu \mathrm{m}$ 的团聚体; A3, 250-2 $000 \mu \mathrm{m}$ 的团聚体; $\mathrm{A} 4,>2000$ $\mu \mathrm{m}$ 的团聚体。

Lowercase letters represent the differences of aggregate fraction in the same soil layer among three types of grassland at the level of $5 \%$. A1, <53 $\mu \mathrm{m}$ aggregate fraction; A2, 53-250 $\mu \mathrm{m}$ aggregate fraction; A3, 250-2 $000 \mu \mathrm{m}$ aggregate fraction; A4, > $2000 \mu \mathrm{m}$ aggregate fraction.

附录II 土层深度和草原类型对团聚体质量百分数的双因素方差分析

Appendix II The two-way ANOVA results of soil depth and grassland type on the mass percentage of aggregate

\begin{tabular}{lc}
\hline 因素 Factor & 团聚体质量百分数 Mass percentage of aggregate \\
\hline 土层深度 Soil depth & 0.12 \\
草原类型 Grassland type & $<0.01$ \\
土层深度×草原类型 Soil depth $\times$ Grassland type & 0.41 \\
\hline
\end{tabular}

附录III 各个土层团聚体的平均质量直径与几何平均直径(平均值土标准误差)

Appendix III The mean mass diameter and geometric mean diameter of soil aggregates in different soil layers (mean $\pm S E$ )

\begin{tabular}{|c|c|c|c|c|c|c|}
\hline \multirow[t]{2}{*}{ 土层 Soil layer $(\mathrm{cm})$} & \multicolumn{2}{|c|}{ 草甸草原 Meadow steppe } & \multicolumn{2}{|c|}{ 典型草原 Typical steppe } & \multicolumn{2}{|c|}{ 荒漠草原 Desert steppe } \\
\hline & $M M D$ & $G M D$ & $M M D$ & $G M D$ & $M M D$ & GMD \\
\hline $0-10$ & $0.76 \pm 0.11$ & $0.38 \pm 0.10$ & $0.49 \pm 0.04$ & $0.20 \pm 0.02$ & $0.31 \pm 0.02$ & $0.04 \pm 0.01$ \\
\hline $10-20$ & $0.65 \pm 0.09$ & $0.29 \pm 0.07$ & $0.46 \pm 0.03$ & $0.17 \pm 0.02$ & $0.36 \pm 0.02$ & $0.12 \pm 0.01$ \\
\hline $20-30$ & $0.46 \pm 0.06$ & $0.17 \pm 0.03$ & $0.44 \pm 0.03$ & $0.16 \pm 0.02$ & $0.39 \pm 0.03$ & $0.14 \pm 0.01$ \\
\hline $30-50$ & $0.46 \pm 0.05$ & $0.17 \pm 0.02$ & $0.38 \pm 0.02$ & $0.14 \pm 0.01$ & $0.38 \pm 0.01$ & $0.13 \pm 0.01$ \\
\hline $50-70$ & $0.43 \pm 0.02$ & $0.16 \pm 0.01$ & $0.33 \pm 0.04$ & $0.12 \pm 0.02$ & $0.39 \pm 0.03$ & $0.13 \pm 0.01$ \\
\hline $70-100$ & $0.37 \pm 0.05$ & $0.12 \pm 0.03$ & $0.31 \pm 0.03$ & $0.11 \pm 0.01$ & $0.40 \pm 0.05$ & $0.14 \pm 0.03$ \\
\hline
\end{tabular}

$G M D$, 几何平均直径; $M M D$, 平均质量直径。

$G M D$, geometric mean diameter; $M M D$, mean mass diameter.

www.plant-ecology.com 


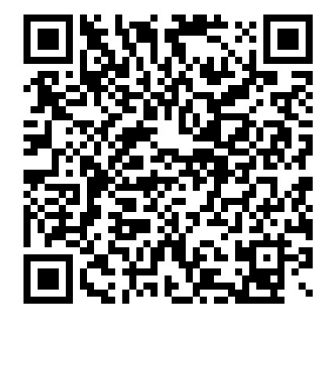

